\title{
Fluorescence polarization imaging of Sisyphus cooling in an atomic beam
}

\author{
B. M. Sparkes, ${ }^{1}$ K. P. Weber, ${ }^{1}$ C. J. Hawthorn, ${ }^{1}$ M. R. Walkiewicz, ${ }^{1}$ E. J. D. Vredenbregt, ${ }^{2}$ and R. E. Scholten ${ }^{1, *}$ \\ ${ }^{1}$ School of Physics, University of Melbourne, VIC 3010, Australia \\ ${ }^{2}$ Department of Applied Physics, Eindhoven University of Technology, 5600MB The Netherlands
}

(Received 4 June 2015; published 11 August 2015)

\begin{abstract}
Sisyphus cooling plays an important role in many cold atom applications including the formation of BoseEinstein condensates and collimation of atomic beams. Here we present a method for measuring the localization of atoms by monitoring the polarization of fluorescence, providing a quantitative two-dimensional image of the cooling process. We outline the concept and provide theoretical models for the optical pumping, cooling, and channeling mechanisms, and present experimental data revealing the development of strong fluorescence polarization in an atomic beam during transverse (lin $\perp$ lin) cooling.
\end{abstract}

DOI: 10.1103/PhysRevA.92.023411

PACS number(s): 37.10.De, 37.10.Gh, 32.50.+d

\section{INTRODUCTION}

The Sisyphus polarization-gradient laser cooling scheme is widely employed in magneto-optical traps to reduce the temperature [1-3] and increase the atomic phase-space density [4] of Doppler-cooled atoms prior to evaporative cooling and Bose-Einstein condensation [5]. Sisyphus cooling can also be used to collimate a thermal atom beam [6], with applications in nanofabrication [7], frequency standards in atomic clocks [8], and focused ion beams [9-11].

Optimal Sisyphus cooling depends on many experimental parameters including the laser detuning, intensity, and polarization, the laser-atom interaction time, and ambient magnetic field. The cooling effect is typically characterized by measurement of the external motion of the atomic sample, such as the momentum distribution, density, or beam collimation [6]. Here we consider the polarization of the resonance fluorescence, in particular, the localization of atoms into subregions of the optical field and the evolution of the internal atomic states through optical pumping. The fluorescence polarization thus provides insight into the details of the cooling process and also a quantitative and noninvasive diagnostic.

The general concept of Sisyphus cooling is illustrated in Fig. 1, for an archetypal $F=1 \rightarrow F=2$ transition. Atoms are cooled by a light field composed of two counterpropagating beams with orthogonal linear polarizations (denoted lin $\perp$ lin), which together form a standing wave with spatially varying polarization. The field has position-dependent energy potentials for the different internal states of atoms in the field. If the detuning of the lasers is chosen correctly, the atom is more likely to absorb photons at high potential and decay to lower potential, and over many absorption and spontaneous emission cycles, the atom will lose its kinetic energy and become localized within a potential well [2].

If Sisyphus cooling is applied to an atomic beam transverse to the direction of propagation, then the atoms will be channeled into the potential wells as they proceed through the cooling region. A variety of measurement techniques have successfully demonstrated channeling effects, for example, using spectroscopic methods [12-14], with localization as small as $\lambda / 15$ (rms) for $\mathrm{Rb}$ atoms in a two-dimensional optical lattice [15]. Bragg diffraction [16] and magnetic

\footnotetext{
*scholten@unimelb.edu.au
}

resonance imaging techniques have also been used [17]. These techniques, however, require additional lasers and sample the atoms at only one location.

The atoms scatter photons during the cooling and localization process, and the polarization of the fluorescence depends on the external light field polarization. The polarization of the fluorescence also depends on the internal state of the atoms, which evolves through optical pumping processes, which in turn depend on the external field polarization. The polarization of the cooling laser field is position-dependent, and thus the fluorescence depends on the spatial localization of the atoms. Strongly polarized fluorescence is expected for atoms tightly confined within the $\sigma^{ \pm}$-polarization channels.

Polarization imaging of the fluorescence thus provides a new means of monitoring evolution of the cooling and channeling process. In particular, for one-dimensional transverse cooling of a rubidium atomic beam, the confinement is related to the beam collimation, and fluorescence polarization analysis can be used to optimize the atomic beam brightness. Fluorescence polarization analysis produces a two-dimensional image of the cooling region, with temporal information on the cooling process and without additional optical fields.

In this paper we outline the mechanism behind fluorescence polarization imaging. We calculate the density matrices expected for atoms at different locations in the lin $\perp$ lin field and the expected values for the fluorescence polarization. The model is verified experimentally and demonstrated as a method for determining the temperature of atoms by comparing line polarization with knife-edge transverse momentum divergence measurements.

\section{FLUORESCENCE POLARIZATION}

The relationship between the polarization properties of resonance fluorescence and the internal state of atoms in polarized near-resonant laser fields have been analyzed in detail for use in atomic collision experiments $[18,19]$. The linear polarization of the fluorescence perpendicular to the cooling laser beams is both readily accessible experimentally and sensitive to the effects of localization and optical pumping.

A schematic representation of the experimental arrangement is shown in Fig. 2, using rubidium-85 with cooling on the $D_{2}$ line at $780 \mathrm{~nm}$. The relevant ${ }^{85} \mathrm{Rb}$ level structure is shown in Fig. 3. The natural line width for the $D_{2}$ transition is 


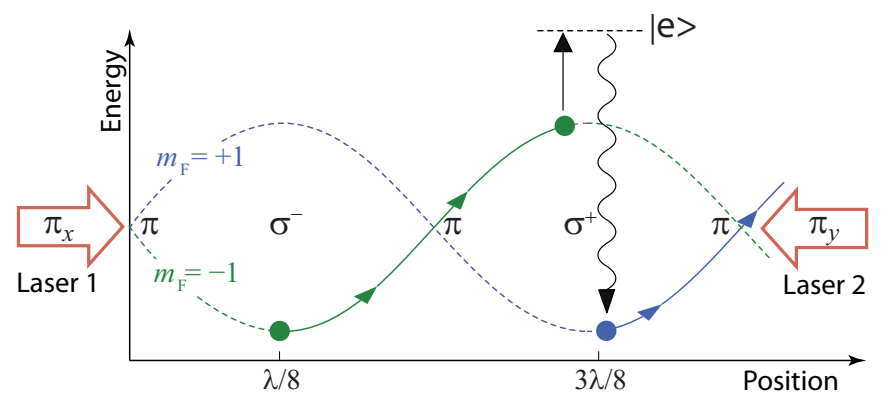

FIG. 1. (Color online) The interference of two orthogonally polarised, counterpropagating lasers leads to a position-dependent polarization seen by atoms and therefore differences in the internal energy levels of the ground states $m_{F}= \pm 1$, for an $F=1 \rightarrow F=2$ transition. At an energy maxima an atom is more likely to absorb a photon and pass, via an excited state $|e\rangle$, to the lower-energy ground state.

$\Gamma=6.07 \mathrm{MHz}$ with saturation intensity $I_{\text {sat }}=1.67 \mathrm{~mW} \mathrm{~cm}^{-2}$ [20]. The light field is tuned slightly below the $\lambda=780 \mathrm{~nm}$ $5^{2} S_{1 / 2} F=3 \rightarrow 5^{2} P_{3 / 2} F=4$ cycling transition.

The line polarization of the fluorescence is defined as

$$
P_{L}=\frac{I_{\|}-I_{\perp}}{I_{\|}+I_{\perp}},
$$

where $I_{\|} \equiv I\left(\alpha=0^{\circ}\right)$ and $I_{\perp} \equiv I\left(\alpha=90^{\circ}\right)$ represent the fluorescence detected with a linear polarization analyzer at an angle $\alpha$ with respect to the laser propagation axis (Fig. 2). $P_{L}$ is therefore measured by taking two fluorescence images through a linear polarization analyzer, rotated by $90^{\circ}$ between each image.

\section{A. Atomic state and fluorescence polarization}

The intensity and polarization of fluorescence depend on the internal state of the atom, which can be conveniently defined in
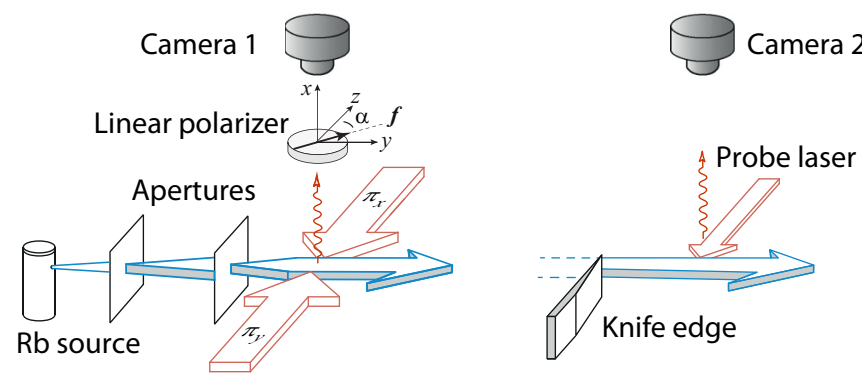

Cooling laser beams

$$
\longmapsto 91 \text { ' } 187 \text { 51 } \quad \text { 90 }
$$

FIG. 2. (Color online) Fluorescence polarization imaging setup, with an atom beam collimated by two counterpropagating laser beams. The fluorescence $\mathbf{f}$ is measured in direction $x$, with polarization direction relative to the cooling laser beam axis $z$. The linear polarization is $I_{\|}\left(I_{\perp}\right)$ when $\alpha=0^{\circ}\left(90^{\circ}\right)$ relative to the $z$ axis. In the experiment, hot ${ }^{85} \mathrm{Rb}$ atoms are emitted from a recirculating candlestick source and initially collimated with two apertures. Sisyphus cooling is then applied and the cooling measured using the linear polarizer and fluorescence imaging camera (1), or a knife edge, a probe laser, and another camera (2).

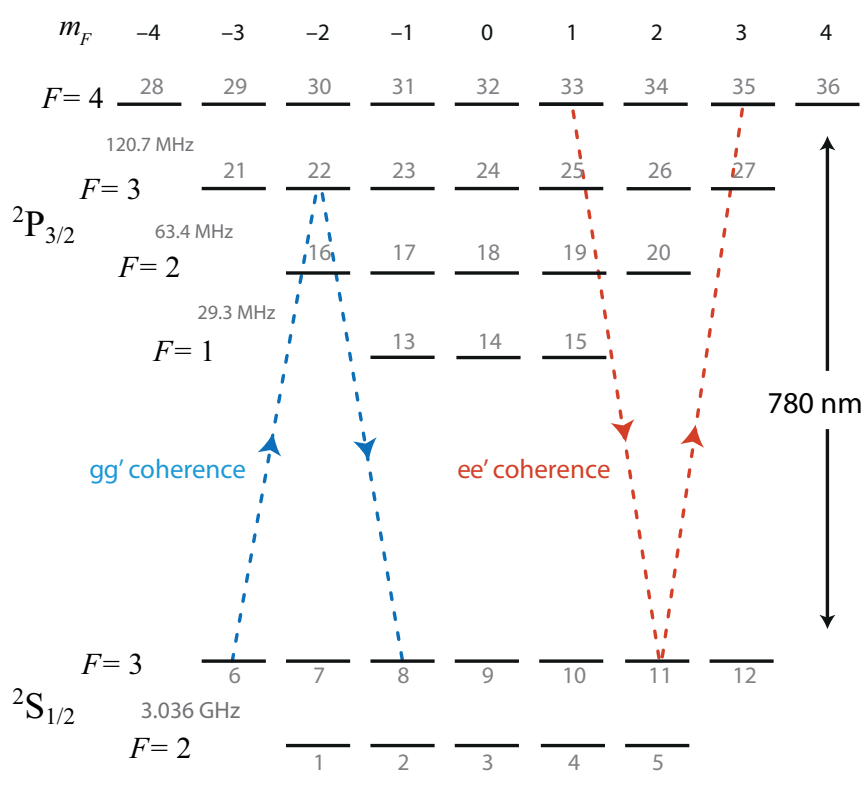

FIG. 3. (Color online) $\mathrm{Rb}^{85}$ energy level structure [21].

terms of the atomic density matrix $\rho$. The detected fluorescence intensity is

$$
I \propto \operatorname{Tr}\{\rho \widehat{F}\}
$$

where $\widehat{F}$ is the fluorescence emission operator. This operator represents a spontaneous emission process from excited state to ground, given by [22]

$$
\widehat{F}=\sum_{g}(\mathbf{f} \cdot \widehat{\mathbf{D}})|g\rangle\langle g|(\mathbf{f} \cdot \widehat{\mathbf{D}})^{\dagger},
$$

where $|g\rangle$ is the ground state of the detected fluorescence transition, $\mathbf{f}$ is a unit vector defined by the polarization axis of the analyzer, and $\widehat{\mathbf{D}}$ is the electric dipole operator. These vectors are expressed in terms of a spherical basis set defined by the general Cartesian unit vectors [23]

$$
\mathbf{e}_{-1}=\frac{1}{\sqrt{2}}(\mathbf{x}-i \mathbf{y}) ; \quad \mathbf{e}_{0}=\mathbf{z} ; \quad \mathbf{e}_{+1}=-\frac{1}{\sqrt{2}}(\mathbf{x}+i \mathbf{y}) .
$$

$\widehat{\mathbf{D}}$ is an irreducible tensor operator of rank 1 and may be expressed in the spherical basis set as

$$
\widehat{\mathbf{D}}=\sum_{q=-1}^{1}(-1)^{q} \widehat{D}_{q} \mathbf{e}_{-q} .
$$

$\mathbf{f}$ is always perpendicular to the direction of observation and, for the particular geometry of our experiments (Fig. 2), can be written explicitly as

$$
\mathbf{f}(\alpha)=\mathbf{y} \sin \alpha+\mathbf{z} \cos \alpha,
$$

where $\alpha$ is the angle of the polarizer axis with respect to the quantization axis used to define the density matrix $\rho$, which we take to be along $z$, the cooling laser axis.

We combine Eqs. (2) and (3) to obtain $I(\alpha)=$ $\sum_{e^{\prime}, e} \rho_{e^{\prime}, e} F_{e, e^{\prime}}(\alpha)$, where the elements $\rho_{e^{\prime} e}$ are expectation values of the slowly varying atomic density operator and $e, e^{\prime}$ index of the excited states. Combining Eqs. (3)-(5), the 
elements of the fluorescence emission operator are given by [19]

$$
\begin{aligned}
F_{e, e^{\prime}}(\alpha)= & \sum_{g}\left\{\left[\sum_{q=-1}^{1}(-1)^{q}\left\langle e\left|D_{q}\right| g\right\rangle \mathbf{f}(\alpha) \cdot \mathbf{e}_{-q}\right]\right. \\
& \left.\times\left[\sum_{q=-1}^{1}\left\langle g\left|D_{q}\right| e^{\prime}\right\rangle \mathbf{f}^{*}(\alpha) \cdot \mathbf{e}_{q}\right]\right\} .
\end{aligned}
$$

Given the atomic density matrix, these matrix elements allow us to predict the polarization of the spontaneous emission using Eq. (2).

\section{ATOMIC STATE CALCULATION}

Theoretical prediction of the line polarization requires detailed knowledge of the atomic density matrix. Optical Bloch equations can be used to predict the atomic state for a given field polarization.

\section{A. Modeling the light field}

The unit polarization vector of the light field can be written as

$$
\boldsymbol{\varepsilon}_{k_{L}}=\frac{1}{\sqrt{2}}[\cos \phi \mathbf{x}+i \sin \phi \mathbf{y}],
$$

where $\mathbf{x}$ and $\mathbf{y}$ are the unit vectors perpendicular to the cooling axis. Any elliptical polarization can be constructed by varying the angle $\phi$.

The generalized Rabi frequency for an arbitrary transition from $|g\rangle \rightarrow|e\rangle$ is [24]

$$
\Omega_{e g}^{k_{L}}=\sqrt{\frac{3 \lambda^{3} \Gamma}{2 \pi h c}} I^{\frac{1}{2}} \mathcal{C}_{e g}^{k_{L}}\left(\boldsymbol{\varepsilon}_{k_{L}} \cdot \mathbf{e}_{k_{L}}\right),
$$

where $\mathcal{C}_{e g}^{k_{L}}$ is the Clebsch-Gordan coefficient for the $g \rightarrow e$ transition with light polarization $k_{L}$. Upon substituting Eq. (8) into Eq. (9), a generalized expression for the Rabi frequency for arbitrary polarized radiation is obtained:

$$
\begin{gathered}
\Omega_{e g}^{ \pm 1}=\sqrt{\frac{3 \lambda^{3} \Gamma}{2 \pi h c}} I^{\frac{1}{2}} \mathcal{C}_{e g}^{ \pm 1}(\cos \phi \pm \sin \phi), \text { for } \Delta m= \pm 1 \\
\Omega_{e g}^{0}=0, \text { for } \Delta m=0 .
\end{gathered}
$$

Thus the atom coupling to the elliptical field is written in terms of separate left- and right-hand circularly polarized components only.

\section{B. The generalized optical Bloch equations}

To calculate the internal state of the atoms in an external field with arbitrary elliptical polarization, we use a full multilevel optical pumping model. We employ the formalism of Refs. $[25,26]$ to accurately describe single-mode radiation excitation of the multiple state transitions required for ${ }^{85} \mathrm{Rb}$ (see Appendix A). In the rotating wave approximation, the generalized optical Bloch equations (GOBEs) require a $36 \times 36$ density matrix for ${ }^{85} \mathrm{Rb}$ in an elliptically polarized field, reducing to 666 simultaneous equations using the hermiticity of the density matrix and neglecting coherences between the lower ground states. Numerical integration of the
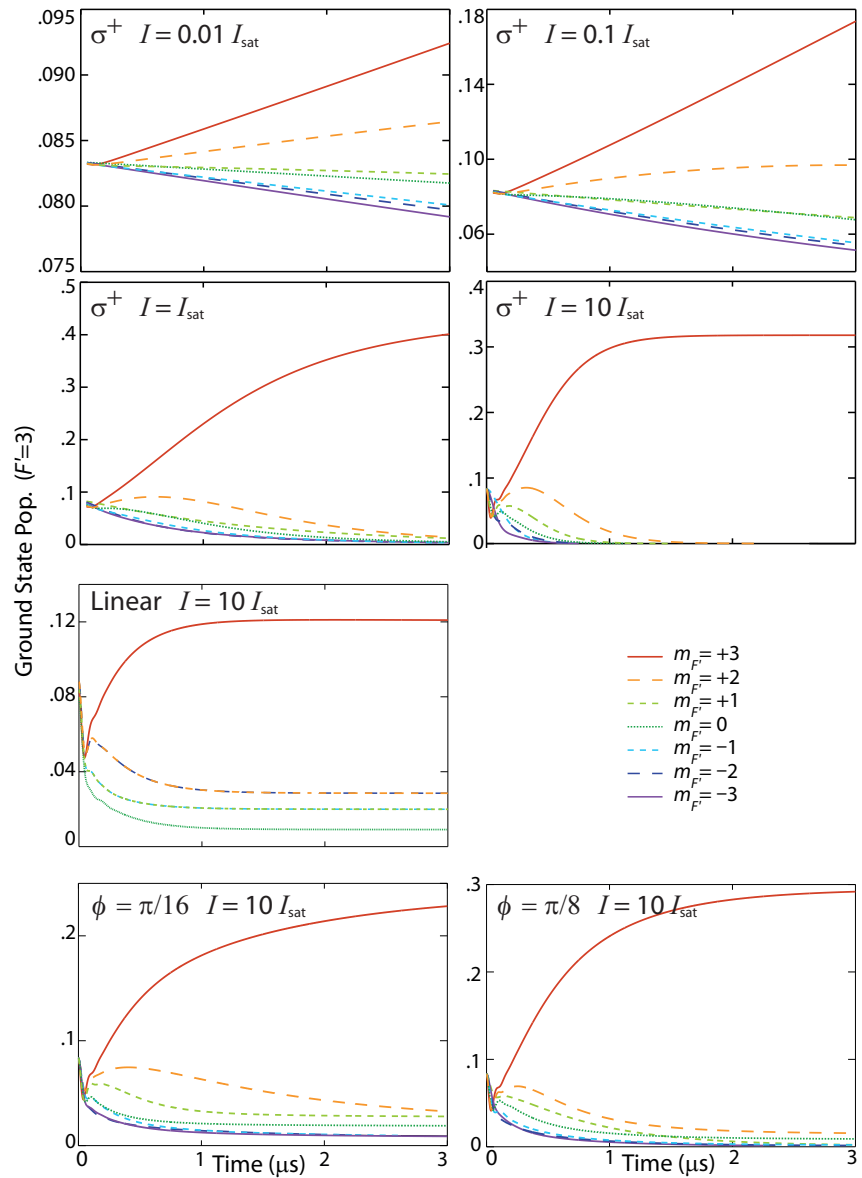

FIG. 4. (Color online) Evolution of the atomic ground state $F=$ 3 populations for a range of intensities $I$, for $\sigma^{+}$, linear (equal $\sigma^{+}$and $\sigma^{-}$), and elliptical $\phi=\pi / 16, \pi / 8$ polarizations. Detuning $\Delta=0$ in all cases.

time-dependent simultaneous linear differential equations then provides $\rho$ for an atom stationary at any location within the lin $\perp$ lin light field (Appendix A).

\section{Atomic state: Simulation results}

\section{Circularly polarized light}

We begin by simulating the time evolution of the populations and coherences among the substates for the case of stationary atoms optically pumped by a light field with pure circular polarization $(\phi=\pi / 4,3 \pi / 4)$, corresponding to an infinitely narrow confinement region. Figure 4 depicts the time evolution of the ground and excited state populations on resonance $(\Delta=0)$, at varying intensities ranging from $I=0.01 I_{\text {sat }} \rightarrow 10 I_{\text {sat }}$. For circular polarization $\left(\sigma^{+}\right)$, the majority of atoms are rapidly pumped into the $\mid F=3, m_{F}=$ $3\rangle \leftrightarrow\left|F=4, m_{F}=4\right\rangle$ cycling transition as expected. Some atoms are lost to the ${ }^{2} S_{1 / 2} F=2$ lower ground state, and, therefore, the total population is not conserved, with greater loss for higher intensity as seen by the decrease in $m_{F}=3$ population from $I_{\text {sat }}=1 \rightarrow 10$. 


\section{Linearly polarized light}

For linearly polarized light fields $(\phi=0, \pi / 2)$ atoms are pumped towards both $\left|F=3, m_{F}= \pm 3\right\rangle$ stretched states, as shown in Fig. 4 for $I=10 I_{\text {sat }}$ and $\Delta=0$. Though initially surprising, the linear polarization discussed here is polarized at $90^{\circ}$ to the chosen quantization axis along the light beams and is therefore an equal mixture of $\sigma^{+}$and $\sigma^{-}$-polarized light, rather than the textbook example of optical pumping with linear light where the quantization axis is chosen to be parallel to the light polarization ( $\pi$-polarized).

\section{Intermediate elliptical polarizations}

Figure 4 also shows results for two different elliptical polarization $\phi=\pi / 16, \pi / 8$. The elliptical polarizations simulate the pumping of atoms located between the circular and linear regions of the lin $\perp$ lin light field and are therefore of great importance in evaluating the line polarization achievable for various degrees of atom localization within the $\sigma^{ \pm}$potential wells.

\section{PREDICTED LINE POLARIZATION}

Using the calculations of $\rho$ presented above, Eqs. (1) and (2) predict the expected line polarization for atoms located at different positions within the lin $\perp$ lin light field.

\section{A. Static line polarization}

For the geometry of fluorescence polarization imaging used in Fig. 2, fluorescence intensities expected for each of the two orthogonal polarizer alignments can be found by taking the trace of the density matrix multiplied by the fluorescence operator [Eq. (2)]. For a multilevel atom in a general elliptically polarized light field, even for fixed polarization (that is, assuming the atoms do not traverse the polarization gradients), the simulations are complex (see Ref. [22]). However, the above optical pumping simulations enable us to determine the density matrix for a stationary atom located at any region within the lin $\perp$ lin light field.

From Sisyphus cooling theory we expect that a high degree of collimation (low transverse velocity) will be correlated with strong confinement of the atoms to the circularly polarized regions of the lin $\perp$ lin field, where the atoms will be pumped to high $\left|m_{F}\right|$. Although the atomic state is fully described in a hyperfine basis, the dipole interaction depends only on the orbital angular momentum $\left(L, m_{L}\right)$ components. These "stretched" excited sublevels correspond to $m_{L}= \pm 1$ in the reduced dipole basis, which have toroidal electron probability distributions, oriented with cylindrical symmetry along the $z$ quantization axis. Linearly polarized radiation emitted by such atoms in the direction of our imaging detector will be polarized perpendicular to the $z$ axis. As a consequence, we expect to see higher intensity if the proposed fluorescence analyzer is perpendicular $(\alpha=\pi / 2)$ to the quantization axis. From Eq. (1), this will result in negative observed line polarizations $P_{L}<0$.

Similar calculations of the steady-state line polarization for atoms at various positions within the cooling field are shown in Fig. 5. The line polarization is clearly dependent on the polarization of light seen by the atoms. For the case of an uncollimated atom beam, the atomic populations will

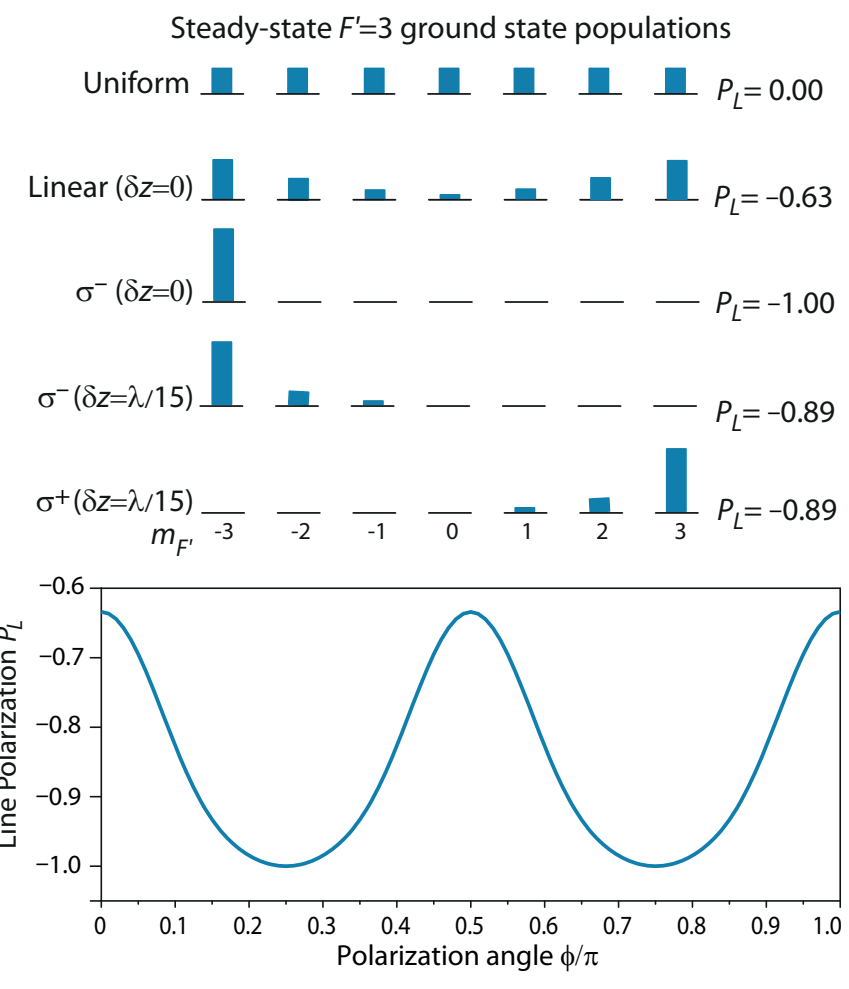

FIG. 5. (Color online) Steady-state populations for different light polarizations with $I=10 I_{\text {sat }}$, and corresponding line polarization $P_{L}$. $\delta z$ indicates the $z$ confinement range of the atoms inside a standing wave. The graph shows the calculated steady-state line polarization as a function of polarization angle $\phi$ assuming $\delta z=0$.

be uniformly distributed among the hyperfine sublevels of the $F=3$ ground and $F=4$ excited states of $\mathrm{Rb}^{85}$, giving $P_{L}=0$ if we take all off-diagonal elements of the density matrix to be zero. A line polarization of $P_{L}=-0.63$ is calculated for atoms situated in the linearly polarized regions of light $(z=0, \lambda / 4)$. The strongest line polarizations of $P_{L}=-1$ will be found for atoms perfectly confined within the circularly polarized regions $(z=\lambda / 8,3 \lambda / 8)$. Figure 5 shows the steady-state line polarization as a function of displacement transverse to the atomic beam, that is, along the $z$ axis of the light field.

For a Gaussian atomic density distribution along $z$,

$$
f(z)=\frac{1}{\pi^{1 / 4}(\delta z)^{1 / 2}} \exp \left[-\frac{1}{2}\left(\frac{z}{\delta z}\right)^{2}\right]
$$

with confinement of the atoms within $\delta z= \pm \lambda / 15$ expected for optimal laser cooling [15], we calculate $P_{L}=-0.89$ would be the strongest line polarization experimentally achievable.

\section{B. Line polarization dynamics}

Though a small fraction of atoms will be highly collimated even before entering the transverse cooling region, most will typically have significant transverse velocities. Due to strong polarization gradients, atoms with any significant transverse velocity will see fluctuating external field polarizations and will not pump into a steady state $\rho$ until cooling confines them within the polarized channels of the field. As the cooling occurs over millisecond time scales [3] and optical pumping 
takes microseconds (see Fig. 4) we expect the evolution of the line polarization to be dominated by the external dynamics of atoms moving through the transverse cooling field.

We can estimate the time scale of cooling and channeling processes from a combination of Doppler cooling and polarization gradient cooling theory. For $|\Delta| \gg \Gamma$, the velocity corresponding to greatest damping force and optimal one-dimensional polarization gradient cooling is given by [3]

$$
v_{c, P G}=\frac{s_{o} \Gamma^{3}}{4 k \Delta^{2}},
$$

where $k=2 \pi / \lambda$ is the wave number and $s_{0}=I / I_{\text {sat }} \cdot v_{c, P G}$ is typically much smaller than the capture velocity for Doppler cooling $v_{c, D}=4.7 \mathrm{~m} / \mathrm{s}$ for ${ }^{85} \mathrm{Rb}$, and Doppler cooling therefore dominates the initial cooling process.

A molasses formed by counterpropagating laser beams of arbitrary intensity results in a force on the atom given by $F=-\beta v$, where $\beta$ is the coefficient of friction given by

$$
\beta=-4 \hbar k^{2} s_{0} \frac{2 \Delta / \Gamma}{\left[1+2 s_{0}+(2 \Delta / \Gamma)^{2}\right]^{2}}
$$

in the case of Doppler cooling for a two-level atom. This leads to a velocity-damping time constant [27]

$$
\tau_{\mathrm{damp}}=-\frac{v}{(d v / d t)_{\mathrm{cool}}}=M / \beta,
$$

where $(d v / d t)_{\text {cool }}=F v=-\beta v^{2}$ and $M$ is the mass of a ${ }^{85} \mathrm{Rb}$ atom. We therefore find $v(t)=v_{0} e^{-\beta t / M}$. The time to cool ${ }^{85} \mathrm{Rb}$ atoms with initial transverse velocities at $v_{c, D}$ to the capture velocity for polarization gradient cooling is then given by

$$
t_{\text {Dcool }}=\frac{-M}{\beta} \ln \left(\frac{v_{c, P G}}{v_{c}}\right) .
$$

For $I=10 I_{\text {sat }}$ and $\Delta=-\Gamma, t_{\text {Dcool }}=500 \mu \mathrm{s}$. Polarization gradient cooling then becomes dominant, and we switch to the much larger friction coefficient given by [27]

$$
\beta_{P G}=-3 \hbar k^{2} \frac{\Delta}{\Gamma}
$$

This again results in an exponentially reducing velocity, with the time required to cool from $v_{c, P G}$ to the recoil velocity $v_{r}=$ $\hbar k / M=6 \mathrm{~mm} \mathrm{~s}^{-1}$ being only $t_{P G \text { cool }}=3 \mu \mathrm{s}$. Thus the polarization gradient cooling time is negligible. The average thermal velocity of atoms in the beam, $\bar{v}=\sqrt{8 k_{B} T / \pi M}=343 \mathrm{~ms}^{-1}$ for the oven temperatures used in the experiment (see Sec. V), with $k_{B}$ the Boltzmann constant. The laser cooling interaction distances can be conveniently described in terms of $L_{\min }=$ $v t_{\text {Dcool }}$, the minimum distance to cool atoms with initial velocity $v$; for example, $L_{\min }=172 \mathrm{~mm}$ for atoms at $343 \mathrm{~m} \mathrm{~s}^{-1}$.

We next adopt a simple qualitative model for the channeling process: a linear interpolation between the case of nonlocalized evenly distributed atoms among all regions of the external field, to that of atoms optimally confined within the polarization channels over a suitable distance in accordance with the cooling time calculated above. We construct a new distribution function $h(y, z)$ as

$$
h(y, z)=a(y) f(z)+[1-a(y)] g(z),
$$

where $y$ is the distance traveled through the interaction region, $f(z)$ is the distribution function for localized atoms defined in

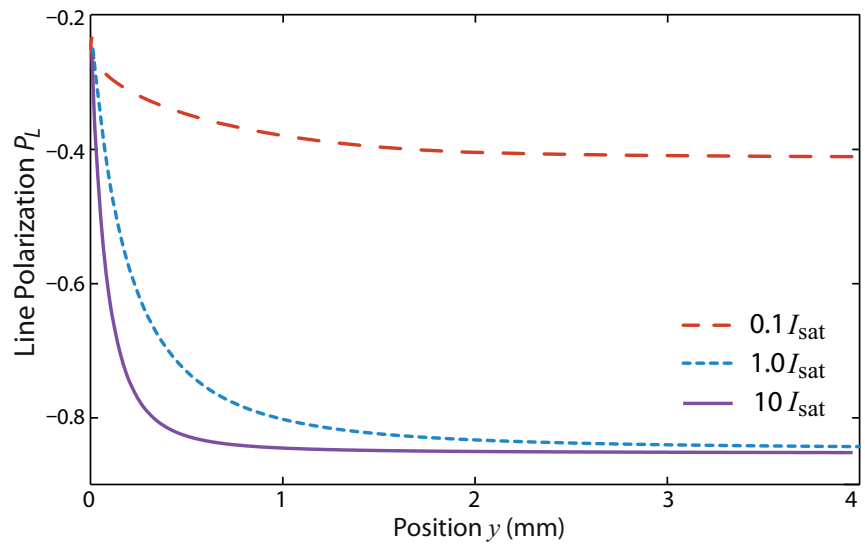

FIG. 6. (Color online) Line polarizations for different intensities as a function of $y$ position through the cooling region for $L_{\min }=$ $150 \mathrm{~mm}(\Delta=-\Gamma)$.

Eq. (12), and $g(z)=1$ represents a uniform distribution along the $z$ axis of the light field. Setting $a(y)=y / L_{\text {min }}$ yields a uniform distribution at the start of the interaction region and a confined distribution function at $y=L_{\min }$. In our experiments the atomic beam is precollimated and $L_{\min }$ is thus much smaller than $172 \mathrm{~mm}$.

The total weighted average line polarization for this distribution,

$$
\left\langle P_{L}(y)\right\rangle_{\text {total }}=\frac{\sum_{y}^{L_{\min }} P_{L}(y) h(y)}{\sum_{y}^{L_{\min }} h(y)},
$$

thus provides a crude estimate for the evolution of line polarization throughout the interaction region, shown in Fig. 6. At high intensities, the minimum line polarization quickly approaches $P_{\mathrm{L}, \min }=-0.85$, but at lower laser intensities the line polarization is still decreasing after $4 \mathrm{~mm}$.

A major shortfall of this model is that it neglects interruptions to the optical pumping process due to the fluctuating polarizations seen by atoms with large transverse velocities. We thus expect the actual time development of line polarization to be slower than predicted by our model. Predictions of the strongest line polarization observable should still be accurate for large interaction distances, as the number of disruptions to optical pumping processes will diminish as atoms become cooled and channeled.

\section{EXPERIMENT}

\section{A. Atom beam and cooling}

The experimental arrangement is shown in Fig. 2. An oven consisting of a recirculating candlestick source surrounded by a copper crucible produces the rubidium beam [28]. The beam is initially collimated by two 1-mm-diameter apertures, one in the candlestick and one in the crucible. A candlestick temperature of $200{ }^{\circ} \mathrm{C}$ produced a beam flux of $3.8 \times 10^{17} \mathrm{~m}^{-2} \mathrm{~s}^{-1}$, measured at a distance of $35 \mathrm{~cm}$ from the candlestick. A 2-mm-diameter aperture at $91 \mathrm{~mm}$ and a $3 \times 1 \mathrm{~mm}^{2}$ rectangular aperture at $187 \mathrm{~mm}$ from the oven were used to provide additional beam collimation. 
The laser cooling was located $51 \mathrm{~mm}$ from the rectangular aperture, and the interaction region was approximately $20 \mathrm{~mm}$ long, determined by the width of the laser beams. The desired lin $\perp$ lin field depicted in Fig. 1 was established from the superposition of the two counterpropagating, orthogonally linearly polarized laser beams. This employed the $5^{2} S_{1 / 2}(F=$ $3) \leftrightarrow 5^{2} P_{3 / 2}(F=4)$ optical cooling transition of ${ }^{85} \mathrm{Rb}$ with a weak repump laser tuned to the $5^{2} S_{1 / 2}(F=2) \leftrightarrow 5^{2} P_{3 / 2}$ $(F=3)$ transition. A repump power of $\approx 1 \mathrm{~mW} \mathrm{~cm}^{-2}$ was found to be sufficient to avoid noticeable loss of atoms to the lower $F=2$ ground state.

\section{B. Conventional divergence measurement: Knife-edge profile}

The fluorescence polarization imaging method was compared to measurements of the atomic beam divergence determined from the spatial derivative of the atomic beam profile [6]. A sharp knife edge was inserted to obscure half of the atomic beam immediately following the laser collimation, and the beam divergence of the cooled beam was obtained from the angular distribution function,

$$
\begin{aligned}
P(\theta) d \theta & \propto\left[\int_{0}^{\infty} d v_{y} v_{y}^{2} \exp \left(\frac{-v_{y}^{2}}{2 v_{0 y}^{2}}\right) v_{z}^{2} \exp \left(\frac{-v_{z}^{2}}{2 v_{0 z}^{2}}\right)\right] d \theta \\
& =2\left(\frac{v_{0 z}^{2} v_{0 y}^{2}}{\theta^{2} v_{0 y}^{2}+v_{0 z}^{2}}\right)^{2} d \theta
\end{aligned}
$$

where $\theta \equiv v_{z} / v_{y}$ is the angle of the atomic velocity vector with respect to the axis of the atomic beam [see Fig. 7(a)], and $v_{y}$ and $v_{z}$ are the longitudinal and transverse velocities, respectively. Velocities $v_{0 y, z}^{2}=k_{B} T_{y, z} / M$ characterize the width of the thermal distributions in the two directions. The spatial profile of the beam may be written as

$$
I(z) \propto \int_{\theta_{\min }}^{\pi / 2} P(\theta) d \theta
$$

where $I(z)$ is the fluorescence intensity at distance $z$ from the beam axis and $\theta_{\min }=\tan ^{-1}(z / L)$, assuming the knife-edge blocks the atomic beam for $z>0$, and $L=1.00 \mathrm{~m}$ is the distance between the knife edge and the fluorescence probe. Taking the derivative of this profile therefore provides a measure of $P(\theta)$.

To find the beam divergence, the spread of the distribution with respect to $\theta$ must be related to the transverse velocity spread $v_{0 z}$. In our case this was found by obtaining a measurement of the full-width-at-half-maximum (FWHM) of the derivative of the fluorescence line profile, and converting it to an angular FWHM, $\theta_{\mathrm{FWHM}}$, by dividing by the separation between knife edge and fluorescence probe. From $P(\theta)$,

$$
\theta_{\mathrm{FWHM}}=2 \sqrt{\sqrt{2}-1} \frac{v_{0 z}}{v_{0 y}} .
$$

$v_{0 y}$ can be calculated from the thermal distribution with $T_{y}=$ $200{ }^{\circ} \mathrm{C}$ so that $v_{0 z}$ can be found from $\theta_{\mathrm{FWHM}}$. The transverse velocity is therefore directly proportional to the FWHM of the derivative of the shadow profile.

The atomic beam was probed with a weak $\left(I_{\text {probe }}=I_{\text {sat }}\right)$ resonant probe beam located $L=1.00 \mathrm{~m}$ downstream from the transverse laser cooling section as shown in Fig. 2. To

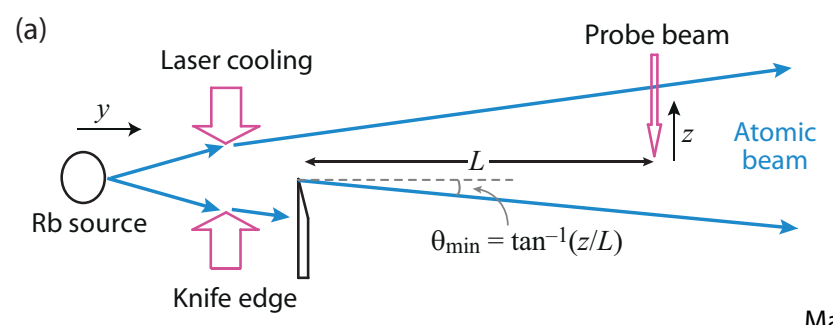

(b)
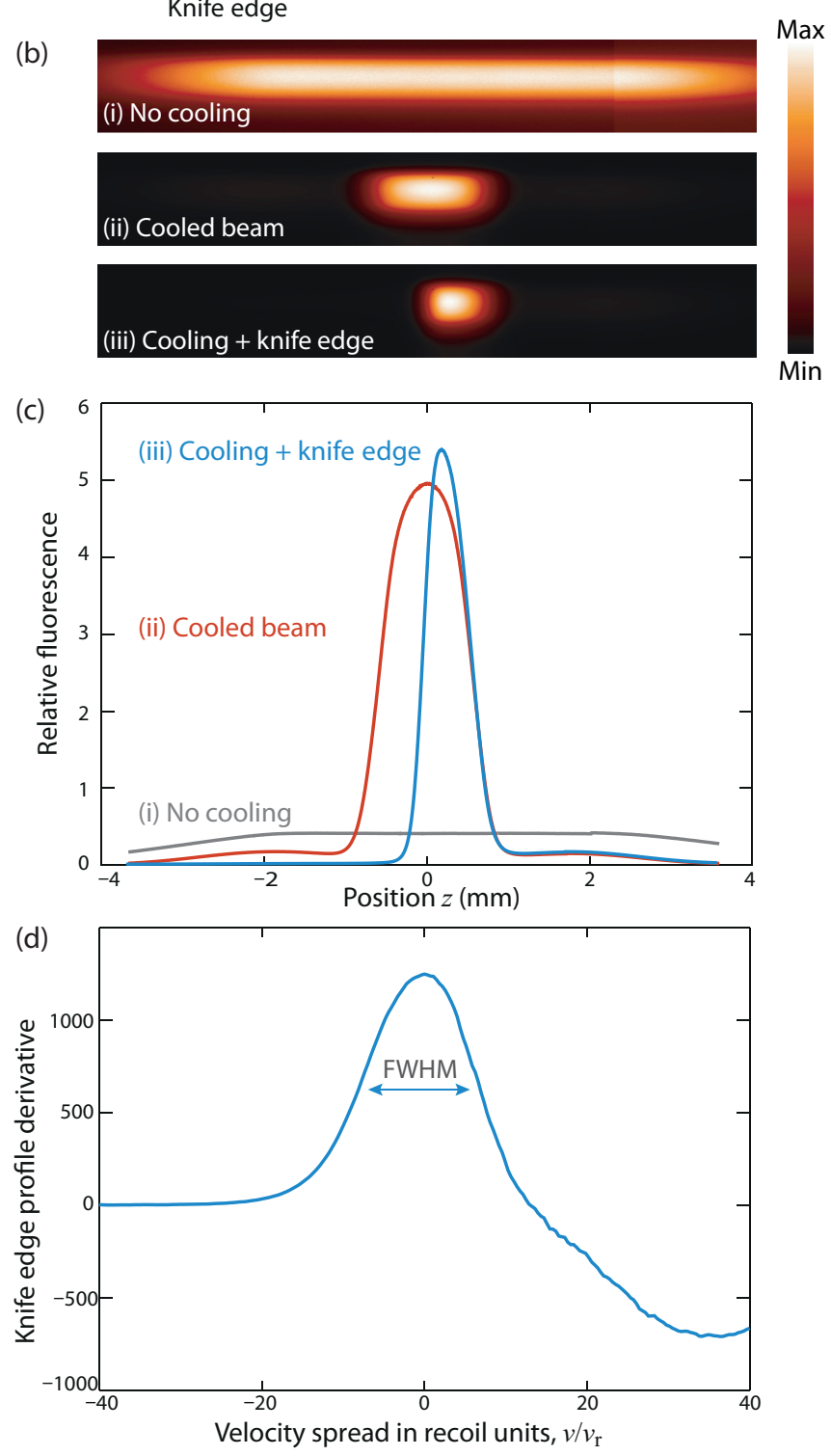

FIG. 7. (Color online) Knife-edge measurements of transverse polarization gradient cooling. Measurement geometry is shown at top, above CCD images and line profiles of the fluorescence intensity from intersection of the atom and probe laser beams, with (i) no transverse cooling, (ii) transverse cooling but no obstruction from the knife edge, and (iii) transverse cooling with knife edge. Bottom graph shows the spatial derivative of the knife-edge line profile.

accurately characterize the effect of transverse laser cooling on beam divergence, and to provide a reference for the central beam position, an image was taken without laser cooling. For each measurement [see Fig. 7(b)], two images of the fluorescence from the probe beam were acquired with camera 

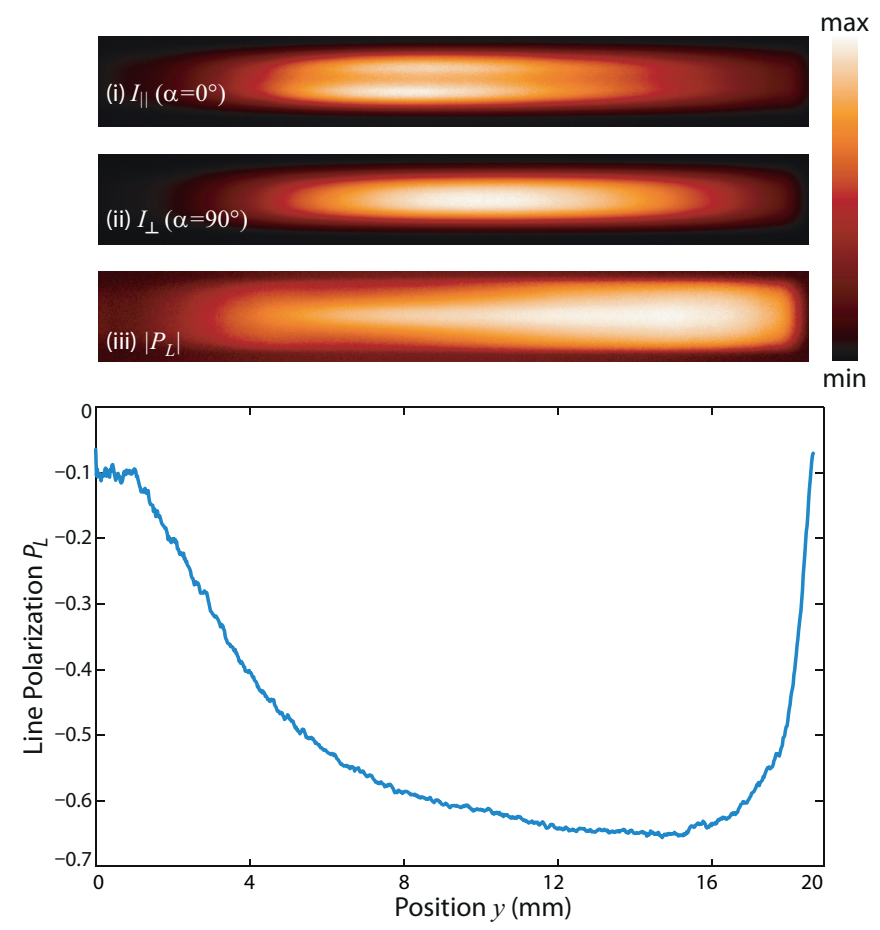

FIG. 8. (Color online) False-color CCD images of fluorescence from the lin $\perp$ lin cooling region of the atom beam, with the polarizer at (i) $\alpha=0^{\circ}$, (ii) $\alpha=90^{\circ}$, and (iii) the resulting $2 \mathrm{D}$ line polarization. The plot shows a line profile through the center region of the polarization.

2: one for the unobstructed atomic beam, and the other with the knife edge inserted into the middle of the beam. No collimation along the $x$ axis was employed, resulting in low atomic density. Exposure times of $10 \mathrm{~s}$ were used to obtain a good signal-to-noise ratio. The derivative of the shadow profile cast by the knife edge was evaluated numerically and the FWHM of the resulting profile used to quantitatively determine beam divergence in units of recoil velocity.

\section{Fluorescence polarization measurement}

Images for polarization analyzer settings parallel $I_{\|}(\alpha=$ $\left.0^{\circ}\right)$ and perpendicular $I_{\perp}\left(\alpha=90^{\circ}\right)$ with respect to the transverse cooling beam at each laser cooling condition were recorded on camera 1 (150 ms exposure). These fluorescence images were then used to determine the line polarization.

Sample images acquired for the two polarizer alignments are shown in Fig. 8 for transverse cooling laser intensity $I=16.8 \mathrm{~mW} \mathrm{~cm}^{-2}\left(10 I_{\text {sat }}\right)$ and detuning $\Delta=-\Gamma$. The two fluorescence images exhibit quite different features as expected, arising from the dipole radiation pattern of the excited $p$ state orbital electron decaying to the spherically symmetric $s$ ground state orbital. Greater fluorescence intensity is seen for polarization perpendicular $\left(\alpha=90^{\circ}\right)$ to the quantization axis defined by the transverse laser cooling beam ( $z$ axis). A dark central region is observed for parallel polarization $\left(\alpha=0^{\circ}\right)$. Atoms in $\sigma^{ \pm}$fields are excited to high $m_{F}$ states with toroidal electron density distributions. These have small dipole moments along the $z$ direction and thus low emission of light polarized along $z$. Cold atoms are channeled into $\sigma^{ \pm}$regions, and thus atoms at the center, which have small transverse momentum, have low emission of light polarized parallel to $\alpha=0^{\circ}$.

A 2D line polarization image is included in Fig. 8, with a profile taken along the center of the atom beam. Two trends are apparent from these results. First, atoms initially exhibit a very weak line polarization upon entering the cooling region, with polarization developing as the atoms traverse the region and are transversely cooled. Second, atoms in the center of the atom beam display the strongest polarizations at all points along the entire length of the interaction region. Both of these trends are consistent with the expected dependence of line polarization upon the spatial confinement of atoms within polarization channels of the field. Localization of atoms into these potential wells is expected to increase as the atoms propagate further into the cooling region and thus exhibit a greater degree of line polarization. Additionally, the component of atoms initially located in the central region of the atom beam will already be well collimated before application of laser cooling. These atoms will immediately have transverse velocities below the capture velocity of polarization gradient cooling and be rapidly cooled and channeled. Atoms with larger initial transverse velocity will be found at the outer edges of the atom beam. They must first undergo Doppler cooling before they can be channeled into regions of constant polarization where optical pumping can approach steady-state alignment. Thus the line polarization at the edges of the atomic beam is initially not as strong.

The minimum line polarization achieved, $P_{L}=-.65$, is an indication of the degree of confinement within the $\sigma^{ \pm}$potential channels. The line polarization rapidly approaches zero as the atoms emerge from the transverse cooling beams and are no longer channeled by the shallow potential wells.

\section{Comparison of different methods}

Altering the cooling laser intensity and detuning will affect the efficacy of the Sisyphus cooling. We can quantify these alterations in terms of the average light shift parameter [6],

$$
U_{o}=\frac{\hbar \Delta}{2} \ln \left(1+\frac{4 \Omega^{2}}{\Gamma^{2}+4 \Delta^{2}}\right),
$$

where $\Omega$ is the Rabi frequency of the cooling lasers. We can therefore compare the line polarization and knife-edge measurement values as $U_{o}$ is varied to see how well they correlate with the transverse temperature of the atom beam. These values are shown in Fig. 9 for a range of light shifts.

For the knife-edge measurements, low intensities and large detunings were found to have the greatest effect in reducing atomic beam divergence, as expected because the velocity capture range for Doppler cooling is proportional to $|\Delta|$. Atoms with small transverse velocities will still be cooled at low detunings and remain in the center of the beam, while those with higher transverse velocities will not be cooled substantially. Large detunings also result in slower Doppler cooling [Eq. (14)] and faster Sisyphus cooling [Eq. (17)]. Line polarization measurements (Fig. 9) show similar behavior, with a linear relationship between $P_{L}$ and $U_{o}$ observed at low detuning, and saturation to $P_{L}=-0.67$ when $\Delta \geqslant \Gamma$. 


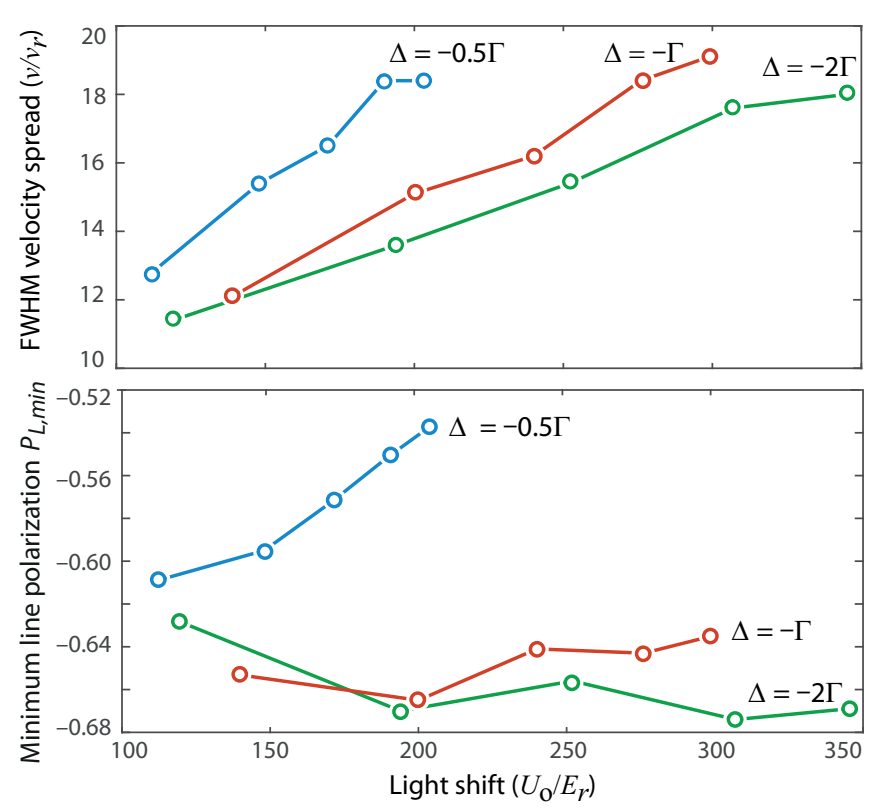

FIG. 9. (Color online) Knife-edge FWHM velocity spread measurements and minimum line polarization measurements as a function of light shift $U_{o}$ normalized to the recoil energy $E_{r}$, for a range of cooling laser detunings $\Delta$.

\section{DISCUSSION}

The experimental results show that the line polarization is correlated with the temperature of the atomic beam, and therefore fluorescence polarization imaging can be a useful tool for monitoring the cooling process. This was seen first as a function of propagation through the cooling regime (Fig. 8) and then as a function of average light shift parameter (Fig. 9) for $\Delta=-\Gamma / 2$.

The line polarization $P_{\mathrm{L} \text {,min }}$ appears to saturate with increasing light shift $U_{o}$, for larger values of detuning $|\Delta|$ and remains above the expected minima of $P_{L}=-0.89$ for confinement within $\pm \lambda / 15$. To investigate further, the cooling conditions were improved for larger detunings, by reducing the laser line widths and expanding the beams to extend the interaction region. With these improvements we found the sensitivity of the fluorescence polarization imaging method improved, with a new minimum value of $P_{L}=-0.74$ (Fig. 10). The divergence measurements indicate that atomic transverse velocities still greatly exceed the recoil velocity, and, therefore, further cooling is required to achieve localization to within $\lambda / 15$ and the optimum $P_{L}=-0.89$.

The dependence of $P_{L}$ along $y$, the atomic propagation direction, shows that cooling is much less rapid than predicted by the theory developed in Sec. IV B. The slower cooling is at least in part due to the Gaussian intensity profiles of the cooling lasers, but also the simplifying assumptions of linear distribution of Eq. (18).

A full quantum Monte Carlo model [29-31] of the cooling process allowed more detailed investigation of the position and time dependence of $P_{L}$. Results of the QMC simulation, described in detail in Appendix A and plotted in Fig. 10, show significantly improved agreement with the experimental results compared to the semiclassical approach

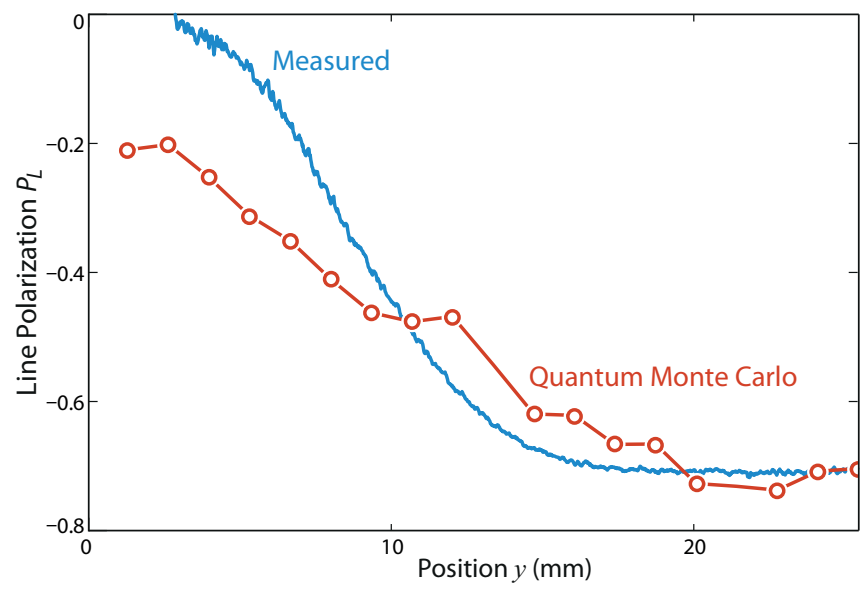

FIG. 10. (Color online) Measured line polarization (blue) and quantum Monte Carlo simulation results (red).

of Fig. 6. As mentioned earlier, the simple semiclassical model is based on an average over steady-state conditions and neglects interruptions to the optical pumping process due to the fluctuating polarization as atoms traverse the external polarization gradient field.

\section{CONCLUSIONS}

Fluorescence polarization imaging provides a new approach to investigating cooling and localization in polarization gradient fields. We have shown how the external field affects the internal atomic state, how cooling and localization of the atoms within defined regions of the polarization gradient field affect the external field experienced by the atoms, and how the fluorescence is affected by the internal atomic state and the external field. Experimental results (Fig. 9) show that the line polarization is correlated to the temperature and confinement of atoms undergoing Sisyphus cooling. This compact diagnostic technique, verified with quantum Monte Carlo simulations, can be used to optimize the cooling and collimation of atomic beams with two-dimensional imaging and without the added complexity of extra optical fields.

\section{ACKNOWLEDGMENTS}

We thank P. M. Farrell and R. P. Anderson for helpful discussions. This work was supported by the Australian Research Council. B.M.S. gratefully acknowledges the support of a University of Melbourne McKenzie Fellowship.

\section{APPENDIX A: GENERALIZED OPTICAL BLOCH EQUATIONS}

The atom-light interaction may be described in terms of the Hamiltonian in the dressed-state picture, given by

$$
\widehat{\mathcal{H}}=\widehat{\mathcal{H}}_{0}+\widehat{\mathcal{H}}_{\text {rad }}+\widehat{\mathcal{H}}_{\text {int }},
$$

where $\widehat{\mathcal{H}}_{0}$ is the field-free, time-independent atomic Hamiltonian with eigenvalues $E_{n} \equiv \hbar \omega_{n}, \widehat{\mathcal{H}}_{\text {rad }}$ is the Hamiltonian of the light field, and $\widehat{\mathcal{H}}_{\text {int }}$ is the atom-field interaction which causes transitions as well as light shifts. 
In the Heisenberg picture, if we assume no explicit time dependence and neglect effects on the external field, the evolution of $\rho$ is given by [32]

$$
\dot{\rho}=-\frac{i}{\hbar}[\rho, \widehat{\mathcal{H}}]=-\frac{i}{\hbar}\left[\rho, \widehat{\mathcal{H}}_{0}\right]-\frac{i}{\hbar}[-\widehat{\mathbf{D}} \cdot \overrightarrow{\mathbf{E}}(\mathrm{t}), \rho]-\widehat{\Gamma}\{\rho\},
$$

where $\overrightarrow{\mathbf{E}}(\mathrm{t})$ is the time-varying electric field connecting the two levels of the atom and $\widehat{\Gamma}$ is an operator describing the radiative relaxation.

Applying the rotating wave approximation to Eq. (A2) results in a set of simplified equations describing the time evolution of the ground and excited state populations, and the off-diagonal coherences, in the presence of spontaneous emission. In the case of a two-level atom, these are known as the optical Bloch equations (OBEs). Generalizing to the case of the ${ }^{85} \mathrm{Rb}$ system (Fig. 3) requires a $36 \times 36$ density matrix, and each of the $36^{2}=1296$ elements evolves according to the appropriate OBE. Exploiting the Hermitian property of the density matrix $\rho_{i j}=\rho_{j i}^{*}$ reduces the problem to 666 independent differential equations. Optical pumping calculations are typically performed for a light field of known circular or linear polarization. For these cases, the relevant dipole selection rules result in a further reduction in complexity that does not occur for arbitrary elliptically polarized light fields. For the elliptical field characterized by angle $\phi$ in Eq. (8), we write the following generalized optical Bloch equations (GOBEs).

Ground state populations $(1 \leqslant g \leqslant 5)$ :

$$
\dot{\rho}_{g g}=\frac{1}{\tau} \sum_{q=-1}^{+1} \sum_{e=13}^{36}\left(\mathcal{C}_{e g}^{q}\right)^{2} \rho_{e e}
$$

Ground state populations $(6 \leqslant g \leqslant 12)$ :

$$
\dot{\rho}_{g g}=-\sum_{e=13}^{36}\left[\Omega_{e g}^{-1}(\cos \phi-\sin \phi)-\Omega_{e g}^{+1}(\cos \phi+\sin \phi)\right] \operatorname{Im}\left\{\rho_{e g}\right\}+\frac{1}{\tau} \sum_{q=-1}^{+1} \sum_{e=13}^{36}\left(\mathcal{C}_{e g}^{q}\right)^{2} \rho_{e e}
$$

Excited state populations $(13 \leqslant e \leqslant 36)$ :

$$
\dot{\rho}_{e e}=\sum_{g=6}^{12}\left[\Omega_{e g}^{-1}(\cos \phi-\sin \phi)-\Omega_{e g}^{+1}(\cos \phi+\sin \phi)\right] \operatorname{Im}\left\{\rho_{e g}\right\}-\frac{1}{\tau} \rho_{e e}
$$

Coherence elements:

$$
\begin{aligned}
\dot{\rho}_{e g}= & -i \Delta_{e g} \rho_{e g}+\frac{i}{2} \sum_{e^{\prime}=13}^{36}\left[\Omega_{e^{\prime} g}^{+1}(\cos \phi+\sin \phi)-\Omega_{e^{\prime} g}^{-1}(\cos \phi-\sin \phi)\right] \rho_{e e^{\prime}} \\
& -\frac{i}{2} \sum_{g^{\prime}=6}^{12}\left[\Omega_{e g}^{+1}(\cos \phi+\sin \phi)-\Omega_{e g}^{-1}(\cos \phi-\sin \phi)\right] \rho_{g^{\prime} g}-\frac{1}{2 \tau} \rho_{e g} \\
\dot{\rho}_{e e^{\prime}}= & -i \Delta_{e^{\prime} e} \rho_{e e^{\prime}}+\frac{i}{2} \sum_{g=6}^{12}\left[\Omega_{e g}^{+1}(\cos \phi+\sin \phi)-\Omega_{e g}^{-1}(\cos \phi-\sin \phi)\right] \rho_{e g} \\
& -\frac{i}{2} \sum_{g=6}^{12}\left[\Omega_{e g}^{+1}(\cos \phi+\sin \phi)-\Omega_{e g}^{-1}(\cos \phi-\sin \phi)\right] \rho_{g e^{\prime}}-\frac{1}{2 \tau}\left(\rho_{e e^{\prime}}+\rho_{e^{\prime} e}\right) \\
\dot{\rho}_{g g^{\prime}}=- & i \Delta_{g^{\prime} g} \rho_{g g^{\prime}}+\frac{i}{2} \sum_{e=13}^{36}\left[\Omega_{e g^{\prime}}^{+1}(\cos \phi+\sin \phi)-\Omega_{e g^{\prime}}^{-1}(\cos \phi-\sin \phi)\right] \rho_{g e} \\
& -\frac{i}{2} \sum_{e=13}^{36}\left[\Omega_{e g}^{+1}(\cos \phi+\sin \phi)-\Omega_{e g}^{-1}(\cos \phi-\sin \phi)\right] \rho_{g^{\prime} e} \\
+ & \sum_{e, e^{\prime}=13}^{36} \sum_{q=-1}^{+1}\left[\Omega_{e^{\prime} g^{\prime}}^{q} \Omega_{e g}^{q} \delta\left(\omega_{\lambda}-\omega_{e}+\omega_{g}\right) \rho_{e e^{\prime}}+\Omega_{e^{\prime} g}^{q} \Omega_{e g^{\prime}}^{q} \delta\left(\omega_{\lambda}-\omega_{e}+\omega_{g^{\prime}}\right) \rho_{e^{\prime} e}\right] .
\end{aligned}
$$

We have separated the elements into four blocks: matrices $\rho_{g g}$ and $\rho_{e e}$ are submatrices for the ground and excited states, and off-diagonal blocks $\rho_{e g}$ and $\rho_{g e}$ describe the optical coherences. $\tau$ is the radiative lifetime of the $5^{2} \mathrm{P}_{3 / 2}$ state $(\tau=1 / \Gamma=25.8 \mathrm{~ns}) . \delta$ is the Dirac delta function. The terms $\Delta_{\alpha \beta}=\omega_{\alpha}-\omega_{\beta}-\omega_{0}$ describe the detuning of a given transition from the applied cooling laser at frequency $\omega_{0}$. The nature of the $g g^{\prime}$ and $e e^{\prime}$ coherence terms is visualized by the 


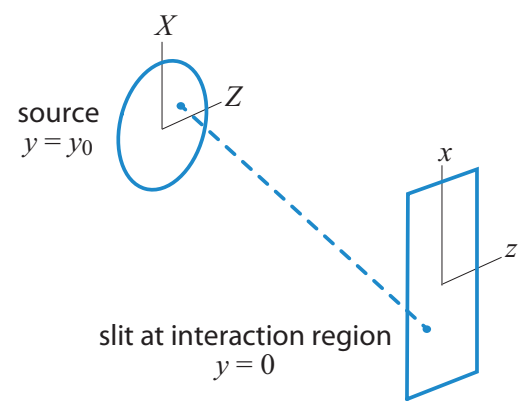

FIG. 11. (Color online) Geometry of source and rectangular slit definining the initial beam prior to transverse cooling.

example transitions depicted in Fig. 3, which involve multiple transitions linking two $m_{F}$ sublevels within one ground or excited hyperfine level. $\rho$ is normalized such that $\operatorname{Tr}\{\rho\}=1$.

A further simplification can be made by assuming the $F=2$ hyperfine ground states will not be excited by radiation tuned to the $F=3 \leftrightarrow F=4$ transition. Atoms spontaneously decaying into the $F=2$ ground state may therefore be neglected in the optical pumping simulation as they will play no further role. This is not the case in experiments where these atoms are transferred back to the $F=3$ states with a repumping laser. The impact of repumping on the relative population distributions for steady-state optical pumping, however, will be negligible, as are the density matrix elements representing coherences between the lower ground states. These assumptions remove a further 165 coherence terms and their complex conjugates. Therefore a full optical pumping calculation for arbitrary elliptically polarized light fields requires the solution of the GOBEs [Eqs. (A3)-(A8)] for just 501 of the original 1296 density matrix elements. A fourth-order Runge-Kutta algorithm was implemented to numerically solve these time-dependent simultaneous linear differential equations.

\section{APPENDIX B: QUANTUM MONTE CARLO SIMULATIONS}

For the quantum Monte Carlo (QMC) simulations we begin with an atom source defined by a circular aperture of radius $R=0.5 \mathrm{~mm}$ at a longitudinal position of $y_{o}=-205 \mathrm{~mm}$, and the beam is collimated by a rectangular slit $(1 \mathrm{~mm} \times 3 \mathrm{~mm})$ at $y=0$ (see Fig. 11). The interaction region extends from $y=0$ to $20 \mathrm{~mm}$. The coordinates at the source are $(X, Z)$ and in the interaction region are $(x, z)$. The transverse velocities are $\left(v_{x}, v_{z}\right)$.

The QMC simulations calculate the rate of emission $\mathcal{R}_{0,90}\left(v_{z}, \tau_{i}\right)$ in the two orthogonal polarization directions $\left(\alpha=0,90^{\circ}\right)$ for an atom that has started at $y=0$ with initial transverse velocity $v_{z}$ and has since spent a time $\tau_{i}$ in the interaction region. The experimental intensity profiles $I_{0,90}(y, z)$ are then given by

$$
I_{0,90}(y, z) \propto \iint d v_{z} d \tau_{i} w\left(v_{z} ; z, y, v_{y}\right) F\left(\tau_{i}\right) \mathcal{R}_{0,90}\left(v_{z}, \tau_{i}\right),
$$

where $w$ is a weighting function accounting for the circular source and rectangular collimation apertures, time $\tau_{i}=y / v_{y}$, and $F\left(\tau_{i}\right)$ is the distribution of interaction times due to the Maxwellian distribution of longitudinal atomic velocities (see Sec. V B). The quantity $\mathcal{R}_{0,90}\left(v_{z}, \tau_{i}\right)$ is the fluorescence rate for an atom that has started at $y=0$ with initial transverse velocity $v_{z}$ and has since spent a time $\tau_{i}$ in the interaction region, for each of the two orthogonal polarization directions $\left(\alpha=0,90^{\circ}\right)$. The fluorescence rate is calculated as $\operatorname{Tr}\{\rho \widehat{F}\}$, which corresponds to the right-hand side of Eq. (2), with $\widehat{F}$ the fluorescence operator defined in Eq. (3). The density matrix $\rho$ follows from the wave function describing the internal and external degrees of freedom of an atom, which is evolved according to the quantum Monte Carlo method described in Refs. [30,33]. The result for $\mathcal{R}$ is averaged over many individual quantum trajectories. The detector integrates along $x$ and hence the weighting function $w$ includes summation of contributions along $x, X$, and $v_{x}$. Since the interaction length is small compared to the distance from the source to the slit, the transverse velocity is already small, and we can assume straight-line motion in the interaction region.

To evaluate Eq. (A1), 12 separate simulations were summed for equally spaced interaction times, chosen to cover $85 \%$ of the integral over the distribution $F(t)$. For each time step $t_{j}$, we consider $N_{j}=N F\left(t_{j}\right)$ atoms, where $\sum_{j} N_{j}=1000, j=$ 1..12. The $N_{j}$ atoms are uniformly distributed over the range of transverse velocities $v_{y}(W+R) /\left(-y_{o}\right)>v_{z}>v_{y}(-W-$ $R) /\left(-y_{o}\right)$, where $v_{y}=Y / \tau_{i}, Y=20 \mathrm{~mm}$ interaction length and $W=0.5 \mathrm{~mm}$ is the slit half-width.
[1] J. Dalibard and C. N. Cohen-Tannoudji, J. Opt. Soc. Am. B 6, 2023 (1989).

[2] C. N. Cohen-Tannoudji and W. D. Phillips, Phys. Today 43(10), 33 (1990).

[3] H. J. Metcalf and P. van der Straten, Laser Cooling and Trapping (Springer-Verlag, New York, 1999).

[4] B. M. Sparkes, J. Bernu, M. Hosseini, J. Geng, Q. Glorieux, P. A. Altin, P. K. Lam, N. P. Robins, and B. C. Buchler, New J. Phys. 15, 085027 (2013).

[5] M. H. Anderson, J. R. Ensher, M. R. Matthews, C. E. Wieman, and E. A. Cornell, Science 269, 198 (1995).

[6] R. E. Scholten, R. Gupta, J. J. McClelland, R. J. Celotta, M. S. Levenson, and M. G. Vangel, Phys. Rev. A 55, 1331 (1997).
[7] J. J. McClelland, R. E. Scholten, E. C. Palm, and R. J. Celotta, Science 262, 877 (1993).

[8] J. Vanier and C. Audoin, Quantum Physics of Atomic Frequency Standards (Hilger, Bristol, 1989).

[9] L. Kime, A. Fioretti, Y. Bruneau, N. Porfido, F. Fuso, M. Viteau, G. Khalili, N. Šantić, A. Gloter, B. Rasser et al., Phys. Rev. A 88, 033424 (2013).

[10] B. Knuffman, A. V. Steele, J. Orloff, and J. J. McClelland, New J. Phys. 13, 103035 (2011).

[11] N. Debernardi, R. W. L. van Vliembergen, W. J. Engelen, K. H. M. Hermans, M. P. Reijnders, S. B. Van der Geer, P. H. A. Mutsaers, O. J. Luiten, and E. J. D. Vredenbregt, New J. Phys. 14, 083011 (2012). 
[12] C. Salomon, J. Dalibard, A. Aspect, H. Metcalf, and C. N. Cohen-Tannoudji, Phys. Rev. Lett. 59, 1659 (1987).

[13] J. E. Thomas, Opt. Lett. 14, 1186 (1989).

[14] P. Verkerk, B. Lounis, C. Salomon, C. N. Cohen-Tannoudji, J. Y. Courtois, and G. Grynberg, Phys. Rev. Lett. 68, 3861 (1992).

[15] P. S. Jessen, C. Gerz, P. D. Lett, W. D. Phillips, S. L. Rolston, R. J. C. Spreeuw, and C. I. Westbrook, Phys. Rev. Lett. 69, 49 (1992).

[16] G. Birkl, M. Gatzke, I. H. Deutsch, S. L. Rolston, and W. D. Phillips, Phys. Rev. Lett. 75, 2823 (1995).

[17] T. A. Savard, S. R. Granade, K. M. O'Hara, M. E. Gehm, and J. E. Thomas, Phys. Rev. A 60, 4788 (1999).

[18] R. E. Scholten, T. Andersen, and P. J. O. Teubner, J. Phys. B 21, L473 (1988).

[19] X.-K. Meng, W. R. MacGillivray, and M. C. Standage, Phys. Rev. A 45, 1767 (1992).

[20] D. A. Steck, http://steck.us/alkalidata, revision 2.1.5, 19 September 2012.

[21] A. Kramida, Y. Ralchenko, and J. Reader, NIST Atomic Spectra Database (National Institute of Standards and Technology, Gaithersburg, MD, 2012), available at http://physics.nist.gov/asd.

[22] T. J. O'Kane, R. E. Scholten, P. M. Farrell, and M. R. Walkiewicz, Phys. Rev. A 59, 4485 (1999).
[23] A. Corney, Atomic and Laser Spectroscopy (Clarendon Press, Oxford, 1977).

[24] P. M. Farrell and W. R. MacGillivray, J. Phys. A 28, 209 (1995).

[25] P. M. Farrell, W. R. MacGillivray, and M. C. Standage, Phys. Rev. A 37, 4240 (1988).

[26] B. T. H. Varcoe, R. T. Sang, W. R. Mac Gillivray, M. C. Standage, and P. M. Farrell, J. Mod. Opt. 46, 787 (1999).

[27] P. D. Lett, W. D. Phillips, S. L. Rolston, C. E. Tanner, R. N. Watts, and C. I. Westbrook, J. Opt. Soc. Am. B 6, 2084 (1989).

[28] M. R. Walkiewicz, P. J. Fox, and R. E. Scholten, Rev. Sci. Instrum. 71, 3342 (2000).

[29] M. R. Doery, E. J. D. Vredenbregt, and T. Bergeman, Phys. Rev. A 51, 4881 (1995).

[30] M. D. Hoogerland, H. F. P. de Bie, H. C. W. Beijerinck, E. J. D. Vredenbregt, K. A. H. van Leeuwen, P. van der Straten, and H. J. Metcalf, Phys. Rev. A 54, 3206 (1996).

[31] R. E. Scholten, T. J. O'Kane, T. R. Mackin, T. A. Hunt, and P. M. Farrell, Aust. J. Phys. 52, 493 (1999).

[32] A. V. Taichenachev, A. M. Tumaikin, V. I. Yudin, and G. Nienhuis, Phys. Rev. A 69, 033410 (2004).

[33] K. Mølmer, Y. Castin, and J. Dalibard, J. Opt. Soc. Am. B 10, 524 (1993). 


\section{University Library}

\section{- M M N E R VA A gateway to Melbourne's research publications}

Minerva Access is the Institutional Repository of The University of Melbourne

Author/s:

Sparkes, BM;Weber, KP;Hawthorn, CJ;Walkiewicz, MR;Vredenbregt, EJD;Scholten, RE

Title:

Fluorescence polarization imaging of Sisyphus cooling in an atomic beam

Date:

2015-08-11

Citation:

Sparkes, B. M., Weber, K. P., Hawthorn, C. J., Walkiewicz, M. R., Vredenbregt, E. J. D. \& Scholten, R. E. (2015). Fluorescence polarization imaging of Sisyphus cooling in an atomic beam. PHYSICAL REVIEW A, 92 (2), https://doi.org/10.1103/PhysRevA.92.023411.

Persistent Link:

http://hdl.handle.net/11343/116520 